\title{
ENZYME ACTIVITY AND DE RITIS RATIO IN ALCOHOLIC AND NON ALCOHOLIC FATTY LIVER PATIENTS BASED ON ULTRASONOGRAPHY
}

Sanju Rawal, ${ }^{1}$ Aakash Shahi, ${ }^{2}$ Narayan Gautam, ${ }^{3}$ Archana Jayan, ${ }^{3}$ Uday Sharma ${ }^{4}$

\begin{abstract}
INTRODUCTION

Fatty liver disease (FLD) refers to a wide clinical and histological spectrum from simple hepatic steatosis to steatohepatitis or cirrhosis, and FLD has been classified as nonalcoholic FLD (NAFLD) and alcoholic FLD (AFLD) based on etiology and ultrasonography (USG).
\end{abstract}

\section{MATERIAL AND METHODS}

This Cross-sectional study was undertaken in the Department of Radiology, Universal College of Medical Sciences (UCMS), Bhairahawa, Nepal from March 2019 to February 2020. A total of 100 subjects involved where ultrasonographically graded for fatty liver and enzyme activity were assessed to observe their association.

\section{RESULTS}

In 100 cases, 66\% were male and 34\% were female. Ultrasonography (USG) guided FLD grade 1 patients were $81 \%$, FLD grade 2 patients were 19\% where as no cases was observed for FLD grade 3. Fifty Six percent (56\%) of the total cases presented with AFLD while remaining 44\% with NAFLD. There was significant difference in serum glutamate oxaloacetate transaminase (SGOT) (p-value: 0.003), serum glutamate pyruvate transaminase (SGPT) (p-value: 0.011 ) and alkaline phosphatase (ALP) (p-value: 0.003) in AFLD and NAFLD. However, there was no significant association of enzyme activity with FLD grade (p-value $>0.05$ ). There was significant difference in SGOT (p-value: 0.004), SGPT (pvalue: 0.025 ) between AFLD grade 1 (AFLDG1) and NFLD grade 1 (NAFLDG1), SGOT (p-value: 0.016) between AFLDG2 and NAFLDG2 and ALP (p-value: 0.01) between AFLDG1 and NAFLDG1. However, De Ritis ratio was not significantly associated with fatty liver disease.

\section{CONCLUSION}

USG is a non-invasive simple tool for early detection of fatty liver in asymptomatic patients enabling clinicians to achieve early detection in conjunction with hepatic enzymes.

KEYWORDS Alcoholic, Non Alcoholic, De Ritis ratio, Enzyme activity, Fatty liver, Ultrasonography

1 Department of Radiology, Universal College of Medical Sciences, Bhairahawa, Nepal

2 Department of Medicine, Universal College of Medical Sciences, Bhairahawa, Nepal

3 Department of Biochemistry, Universal College of Medical Sciences, Bhairahawa, Nepal

4 Bsc. MLT Final Year Student, Universal College of Medical Sciences, Bhairahawa, Nepal

DOI: http//doi.org/10.3126/jucms.v8i1.29808

For Correspondence

Dr. Sanju Rawal,

Department of Radiology

Universal College of Medical Sciences

Bhairahawa, Nepal

Email : sanjurawalchhetri1983@gmail.com 


\section{INTRODUCTION}

Ultrasonography (USG) is a very efficient and widely available technique for the detection of fatty liver. The overall sensitivity and specificity of ultrasound in detection of moderate to severe fatty liver have been shown to be accurate and comparable to gold standard test liver biopsy. ${ }^{1-3}$ Fatty liver in non-alcoholic individuals is rapidly becoming a major public health challenge. ${ }^{4}$ Severe complications can occur because of the risk of progression to more advanced stages making early non invasive detection of fatty liver disease by USG very clinically important..$^{2-5}$ Alcoholic liver disease (ALD), induced by excessive alcohol consumption, and non alcoholic fatty liver disease (NAFLD), caused by obesity and insulin resistance are the most common diseases associated with hepatic steatosis. ${ }^{6}$

Under invasive procedures comes liver biopsy and noninvasive includes radiological tests like USG and various biochemical tests serum glutamate oxaloacetate transaminase (SGOT), serum glutamate pyruvate transaminase (SGPT), alkaline phosphatase (ALP)., USG which is non-invasive, simple tool, can be used for the early detection of NAFLD in asymptomatic patients and is the single best tool in the evaluation of focal liver lesions, unbeaten by any other imaging modality, due to real-time, dynamic nature, high resolution and good safety record. ${ }^{9}$ The survival of patients with ALD and NAFLD depends on various disease-associated conditions. $^{10}$

This present hospital-based study was undertaken to evaluate the relation of enzyme activity, De Ritis ratio, haemoglobin and albumin as an indicator of chronic liver disease with fatty liver disease in patients diagnosed based on USG.

\section{MATERIAL AND METHODS}

This cross-sectional study was undertaken in the Department of Radiology with collaboration of Department of Medicine and Department of Biochemistry, Universal College of Medical Sciences (UCMS), Bhairahawa, Nepal from March 2019 to February 2020.

Study population and sample size was determined as follow:

$$
\mathrm{n}=\frac{\mathrm{z}^{2} \mathrm{PQ}}{\mathrm{D}^{2}}
$$

$\mathrm{n}=(1.96)^{2} \times 0.07 \times 0.93 /(0.0025)=100$ where, $\mathrm{n}=$ sample size, $\mathrm{z}=$ critical value $=1.96, \mathrm{P}=$ prevalence of disease $=7 \%$, $\mathrm{Q}=$ without disease (1-P), $\mathrm{D}=$ allowance error (5\%).

AFLD Group comprised of excessive alcohol consumption 40 $\mathrm{g} /$ day in men (20 g/day in women), usually $>5$ years and / or ongoing daily consumption $>80 \mathrm{~g}$ in two weeks.

NAFLD group comprised of history of alcohol consumption or alcohol intake less than $140 \mathrm{~g}$ in men (70 $\mathrm{g}$ in women) on average per week in last 12 months. ${ }^{5}$ Patients who have been diagnosed with fatty liver disease based on USG finding of GE LOGIQ6 PRO ultrasound scanner were included in the study. Ethical approval was taken from Institutional Review Committee with Registration number UCMS/IRC/046/19.

\section{Procedure for patients}

Conventional B-mode USG is the most common technique used to assess the presence of fatty liver in clinical settings and population studies. Fatty liver is diagnosed based on the following ultrasound parameters: parenchymal brightness, liver-to-kidney contrast, deep beam attenuation, bright vessel walls, and gallbladder wall definition. ${ }^{11}$

The patients were mostly examined with real-time sonography, after a 6-8 hour abstaining from food. Mostly supine and right anterior oblique views were obtained. Sagittal, transverse, coronal, and subcostal oblique views were also performed using both a standard abdominal transducer and a higher frequency transducer. In few cases intercostal views were also needed.

\section{USG gradings of Fatty liver/Diffuse hepatic steatosis}

Mild (Grade1): Minimal diffuse increase in hepatic echogenicity with normal visualization of diaphragm and intrahepatic vessel borders.

Moderate (Grade 2): Moderate diffuse increase in hepatic echogenicity with slightly impaired visualization of intrahepatic vessels walls and diaphragm.

Severe (Grade 3): Marked increase in echogenicity with poor penetration of posterior segment of right lobe of liver and poor or no visualization of hepatic vessels and diaphragm. The Patients with other causes of liver disease like viral or alcoholic hepatitis, on drugs therapy or any chemotherapy and patient's age less than one year and greater than 80 years were excluded from this study.

\section{Biochemical test procedure}

Serum was separated from the blood sample of patients diagnosed with fatty liver by the help of USG and the tests were carried out. The samples were subjected for measuring liver enzymes which will include SGPT, SGOT (Rietman's and Frankel's method) and ALP (Kind's and King's method). The Albumin was tested by Bromo Cresol Green (BCG) dye binding method. SGOT: SGPT ratio was calculated as De Ritis 
Ratio. Hemoglobin level was obtained from hematology analyzer (Beckman coulter, DxH 520). All the biochemical assays were done in fully automated Humanalyzer-600, Germany.

All the data from cases were fed in MS Excel (Microsoft office 2007) and then analyzed by Statistical Package for Social Service (SPSS) for window version; SPSS 22, Inc., Chicago, IL). All the data were expressed in terms of percentage frequency, Median and compared by non-parametric tests Chi-Square test, Man Whitney U test, Kruskal Wallis test and Spearman's rho correlation etc. P-value $<0.05$ was considered to be statistically significant.

\section{RESULTS}

Table 1. General characteristics of the study subjects based on USG

\begin{tabular}{|c|c|c|c|c|}
\hline Characteristics & FLD G1 & FLD G2 & Total & p-value \\
\hline Median Age (IQR) in years & $44(21-67)$ & $45(24-66) \quad 45$ & $45(20-80)$ & 0.812 \\
\hline $20-40$ years $n(\%)$ & $37(45.7)$ & $9(47.5)$ & 46 & \\
\hline $41-60$ years $n(\%)$ & $30(76.9)$ & $9(47.4)$ & 39 & 0.384 \\
\hline$>60$ years $n(\%)$ & $14(93.3)$ & $1(5.3)$ & 15 & \\
\hline Gender (Male: Female) & $1.8: 1$ & $5.3: 1$ & $1.94: 1$ & \\
\hline Male n $(\%)$ & $50(61.7)$ & $16(84.2)$ & 66 & 0.063 \\
\hline Female $n(\%)$ & $31(38.3)$ & $3(15.8)$ & 34 & \\
\hline \multicolumn{5}{|l|}{ Alcoholism } \\
\hline Alcoholics n (\%) & $42(51.9)$ & $14(73.7)$ & 56 & 0.084 \\
\hline Non-Alcoholics n (\%) & $39(48.1)$ & $5(26.3)$ & 44 & \\
\hline Median Hemoglobin (Hb) IQR & $10(5.7-14.3)$ & $10(7.1-12.9)$ & $10(3.3-17.7)$ & 0.944 \\
\hline $\mathrm{Hb}(\leq 10 \mathrm{~g} / \mathrm{dl}) \mathrm{n}(\%)$ & $56(69.1)$ & $16(88.2)$ & 72 & 0.188 \\
\hline $\mathrm{Hb}(>10 \mathrm{~g} / \mathrm{dl}) \quad \mathrm{n}(\%)$ & $25(30.9)$ & $3(15.8)$ & 28 & \\
\hline Albumin (Alb) IQR & $3.3(2.8-3.8)$ & $3.2(2.3-5)$ & $3.2(3.2-3.5)$ & 0.419 \\
\hline $\operatorname{Alb}(\leq 3.5 \mathrm{~g} / \mathrm{dl}) \mathrm{n}(\%)$ & $62(76.5)$ & $16(84.2)$ & 78 & 0.468 \\
\hline $\operatorname{Alb}(>3.5 \mathrm{~g} / \mathrm{dl}) \mathrm{n}(\%)$ & $19(23.5)$ & $3(15.8)$ & 22 & \\
\hline
\end{tabular}

Table 1 shows that the median age of the study subjects was 45 years with IQR of 20-80 maximum of the patients being aged between 20-40 years followed by 41-60 and least was more than 60 years. There was no statistical significance in age distribution (p-value: 0.384 ). Male to female ratio is $1.94: 1$ with no statistical significance difference in two groups (pvalue: 0.063 ). The percentage of alcoholic and non-alcoholic patients with grade 1 FLD was $51.9 \%$ and $48.1 \%$ respectively and with grade 2 FLD being $73.7 \%$ and $26.3 \%$ respectively with no statistical significance difference ( $p$-value: 0.084 ). The median hemoglobin concentration is 10 with over all IQR $3.3-17.7 \mathrm{~g} / \mathrm{dl}$.

Among the studied cases, $72 \%$ were having hemoglobin $\leq 10$ $\mathrm{g} / \mathrm{dl}$ and $28 \%$ were having normal $>10 \mathrm{~g} / \mathrm{dl}$ hemoglobin concentration. The median serum albumin level was 3.2 with over all IQR of 3.2-3.5 g/dl. Lower levels of serum albumin was seen in $78 \%$ of the cases, while $22 \%$ had normal levels.
There was no statistical significance in distribution of hemoglobin concentration and serum albumin with different grades of FLD p-value being 0.419 and 0.468 respectively.

Table 2. Status of enzyme activity and De Ritis ratio in the study subjects based on USG

\begin{tabular}{lllllllllll}
\hline Enzyme & Status & \multicolumn{3}{c}{ FLD Type } & Total & p-value & \multicolumn{7}{c}{ FLD Grade Total } & p-value \\
& & Alc. Non Alc. & & \multicolumn{2}{c}{1} & 2 & \\
\hline \multirow{2}{*}{ SGOT } & Normal & 28 & 19 & 47 & 0.003 & 39 & 8 & 47 & 0.78 \\
& High & 16 & 37 & 53 & & 42 & 11 & 53 & \\
\multirow{3}{*}{ SGPT } & Normal & 27 & 20 & 47 & 0.011 & 40 & 7 & 47 & 0.32 \\
& High & 17 & 36 & 53 & & 41 & 12 & 53 & \\
ALP & Normal & 39 & 35 & 74 & 0.003 & 61 & 13 & 74 & 0.530 \\
& Low & 30 & 31 & 61 & & 20 & 6 & 26 & \\
\multirow{5}{*}{ De Ritis } & $<1$ & 17 & 19 & 36 & 0.351 & 31 & 6 & 37 & 0.568 \\
& $>2$ & 12 & 6 & 18 & & 13 & 5 & 18 & \\
& $1-1.99$ & 27 & 19 & 46 & & 37 & 8 & 45 & \\
\hline
\end{tabular}

Table no 2 shows the association of enzyme activities results with alcoholic FLD. There was statistical significant difference in SGOT (p-value: 0.003), SGPT (p-value: 0.011) and ALP (p-value: 0.003). There was no significant association enzyme activity with grade of FLD (pvalue $>0.05)$.

Table 3. Association of enzyme activity, De Ritis ratio, hemoglobin and albumin level with grades of alcoholic and non-alcoholic FLD based on USG

\begin{tabular}{lcccccc}
\hline Lipid & AFLG1 & AFLG2 & NAFLG1 & NAFLG2 & Total & p-value \\
\hline SGOT & $71^{* \mathrm{c}}$ & $207^{* \mathrm{~b}}$ & $40^{* \mathrm{c}}$ & $25^{* \mathrm{~b}}$ & 50 & 0.001 \\
SGPT & $61.5^{* \mathrm{~d}}$ & $77^{* \mathrm{a}}$ & $32^{*^{\mathrm{d}}}$ & $33^{* \mathrm{a}}$ & 41.5 & 0.007 \\
ALP & $263^{* \mathrm{e}}$ & 214 & $22 *^{* \mathrm{e}}$ & 220 & 228 & 0.099 \\
De Ritis & 1.2 & 1.3 & 1.08 & 0.81 & 1.17 & 0.447 \\
Hb & 10 & 9.5 & 9.98 & 10.1 & 10 & 0.954 \\
Alb & 3.3 & 3.2 & 3.2 & 3.1 & 3.2 & 0.790 \\
\hline
\end{tabular}

Man Whitney U test p-value: ${ }^{\text {*a }} 0.004,{ }^{\text {,b }} 0.016,{ }^{\text {"c }} 0.007,{ }^{\text {,d }} 0.025,{ }^{\text {"ec }} 0.01$

There was statistical significant difference in median value for overall groups of SGOT (p-value: 0.001 ) and SGPT (p-value: 0.007). There was statistical significant difference in SGOT (p-value: 0.007), SGPT (p-value: 0.025) between AFLG1 and NAFLG1, SGOT (p-value: 0.016), SGPT (p-value: 0.004) between AFLG2 and NAFLG2, ALP between AFLG1 and NAFLG1. 
Table 4. Association of liver enzymes with hemoglobin and albumin status

\begin{tabular}{|c|c|c|c|c|c|c|c|}
\hline \multirow{2}{*}{$\begin{array}{l}\text { Liver } \\
\text { enzymes }\end{array}$} & & \multicolumn{2}{|c|}{$\mathrm{Hb}$} & \multirow[t]{2}{*}{ p-value } & \multicolumn{2}{|c|}{ Alb } & \multirow[t]{2}{*}{ p-value } \\
\hline & & Normal & Anemic & & Normal & Low & \\
\hline \multirow[t]{3}{*}{ SGOT } & Normal & 14 & 33 & & 13 & 34 & \\
\hline & High & 14 & 39 & 0.70 & 9 & 44 & 0.19 \\
\hline & Total & 28 & 72 & & 22 & 78 & \\
\hline \multirow[t]{3}{*}{ SGPT } & Normal & 12 & 35 & & 13 & 34 & \\
\hline & Low & 16 & 37 & 0.62 & 9 & 44 & 0.19 \\
\hline & Total & 28 & 72 & & 22 & 78 & \\
\hline \multirow[t]{3}{*}{ ALP } & Normal & 18 & 56 & & 15 & 59 & \\
\hline & High & 10 & 16 & 0.16 & 7 & 19 & 0.48 \\
\hline & Total & 28 & 72 & & 22 & 78 & \\
\hline
\end{tabular}

Table 4 shows that the association of enzyme activity with hemoglobin status and albumin status which were statistically non-significant.

\section{Table 5. Spearman's rho correlation of study variables}

\begin{tabular}{lccccc}
\hline Variables & FLD & A/N FLD & De Ritis & Hb & Alb \\
\hline SGOT & 0.113 & $-0.327^{* *}$ & $0.335^{* *}$ & 0.031 & -0.021 \\
& 0.261 & 0.001 & 0.001 & 0.759 & 0.838 \\
\multirow{2}{*}{ SGPT } & 0.102 & $-0.280^{* *}$ & $-0.211^{*}$ & 0.039 & 0.056 \\
& 0.313 & 0.005 & 0.035 & 0.702 & 0.578 \\
\multirow{2}{*}{ ALP } & -0.16 & $-0.225^{*}$ & 0.006 & $0.229^{*}$ & 0.065 \\
& 0.875 & 0.024 & 0.954 & 0.022 & 0.520 \\
\multirow{2}{*}{ De Ritis } & 0.076 & -0.092 & 1.000 & 0.037 & -0.173 \\
& 0.453 & 0.363 & - & 0.718 & 0.085 \\
Hb & -0.007 & 0.054 & 0.037 & 1.000 & -0.026 \\
& 0.944 & 0.597 & 0.718 & - & 0.795 \\
Alb & -0.065 & -0.083 & -0.173 & -.026 & 1.000 \\
& 0.520 & 0.414 & 0.08 & 0.795 & - \\
\hline
\end{tabular}

${ }^{*}$ p-value at $<0.01,{ }^{*}$ p-value at $<0.001$

Table 5 shows the Spearman's correlation of the enzyme activity with different variables. Alcoholic and non-alcoholic FLD have statistically negative correlation with SGOT (pvalue: 0.001), SGPT (p-value: 0.005) and ALP level (p-value: $0.024)$. Serum SGOT and SGPT have significant correlation with De Ritis ratio (p-value of 0.001 and p-value: 0.035 ) respectively. Similarly, ALP shows significant correlation with hemoglobin (p-value: 0.022). As compared with the alcoholic and non-alcoholic fatty liver, the statistical significance was observed with SGOT (p-value: 0.001), SGPT (p-value: 0.005) and ALP (p-value: 0.025).

\section{DISCUSSION}

With the rising prevalence of obesity, the proportion of fatty liver has increased in both Eastern and Western countries. ${ }^{4}$ ALD represents a broad range of histological changes ranging from simple steatosis to heavier forms of liver injury, including alcoholic hepatitis (AH), cirrhosis, or the parallel development of hepatocellular carcinoma (HCC). ${ }^{12}$

NAFLD is emerging as the most common chronic liver condition in the Western world. It is associated with insulin resistance and frequently occurs with features of the metabolic syndrome. Disease presentation ranges from asymptomatic elevated liver enzyme levels to cirrhosis with complications of liver failure and hepatocellular carcinoma. ${ }^{13}$ Mahaling DU et al(2013), in their study out of 70 cases which were diagnosed as NAFLD on USG, grade 1 NAFLD cases were $47.15 \%$, grade 2 were $42.85 \%$ and grade 3 were $10 \%$. The mean age of the patients was found to be 49.14 years. Male to female ratio was 3:4. ${ }^{14}$ In our study, out of 100 cases, $81 \%$ were diagnosed as grade 1 FLD while $19 \%$ as grade 2 FLD. $56 \%$ were AFLD while $44 \%$ were NAFLD. In the present study, the median age of the subjects was 45 years with IQR 20-80 years and male to female ratio was 1.8:1.

Amadol NL et al (2017), in their study found that the most frequent disorder was elevation of ALT levels (72.5\%), higher than AST (25\%) and ALP (45\%). ${ }^{15}$ In our study, SGOT, SGPT and ALP were showed correlation with AFLD and NAFLD but SGOT and ALP showed stronger correlation than ALT with p-value of 0.003 each, unlike their study.

In the study by Bhusal K, Simkhada R, Nepal P (2017), out of total 100 cases, mild nonalcoholic fatty liver disease was found in $83 \%$, moderate in $17 \%$ and severe in none of the participants. Age of the participants ranged from 26 to 79 years with mean being $45 \pm 11.99$ years. Quite similar to their study, $88.6 \%$ of the non-alcoholic patients were having grade 1 (mild) fatty liver disease, $11.4 \%$ of them were having grade 2 (moderate) fatty liver disease and none of the participants had grade 3 (severe) fatty liver disease. ${ }^{16}$ We studied patients with ALD in whom $81 \%$ were having grade I and $19 \%$ had grade II FLD. Mittal A et al in their study found that there was a significant correlation between sex as well as age group with Alcoholic liver disease. The mean $\pm \mathrm{SD}$ value of De Ritis ratio was $1.59 \pm 0.58$ and it was significantly correlated with ALD. ${ }^{17}$ Jwarchan B et al conducted a study in which total 85 patients were enrolled and they found that females (65.88\%) were more affected by NAFLD than males $(34.12 \%)$. The mean \pm SD of only ALT showed statistical significant difference between grade 1 and grade 2 NAFLD $(\mathrm{p}$-value $=0.027) .{ }^{18} \mathrm{In}$ our study, there was no such difference between males and 
females were considered. The median age of the subjects was 45 years with inter quartile range of $20-80$ years. The enzymes SGOT, SGPT and ALP were found to be statistically associated with the type of FLD, p-value being $0.003,0.011$ and 0.003 respectively. However, there was no association observed to exist with De Ritis ratio. When Man Whitney U test was used, there was statistical significant difference in median value in SGOT (p-value: 0.007), SGPT (p-value: 0.025 ) between AFLG1 and NAFLG1, SGOT (p-value: 0.016), SGPT (p-value: 0.004) between AFLG2 and NAFLG2, ALP between AFLG1 and NAFLG1. Ramesh et al in their case control study found that the increase in AST and ALT levels in AFLD were highly significant when compared to controls $(p$-value $=0.0001)$. Similarly, mean De Ritis ratio showed significant correlation with AFLD $(\mathrm{p}$-value $=0.0001)$ while not with NAFLD. ${ }^{19}$ But in our study, there was no correlation found between De Ritis ratio and type or the grade of FLD. But similar to their study, increase in SGOT and SGPT levels were significant with type and grade of FLD.

\section{CONCLUSION}

Although liver biopsy is the gold standard method for diagnosis of fatty liver, it cannot be performed in the entire general population. Ultrasonography, which is a non invasive and simple tool, can be used for the early detection of fatty liver in asymptomatic patients, where symptoms occur only in the advanced stages of the disease. This technique can also serve as an indicator of future comorbidities enabling clinicians to suggest blood investigations like SGOT and SGPT for proper counselling to patients regarding further management or preventive measures.

\section{ACKNOWLEDGEMENTS}

Our sincere thanks to all patients who had undergone USG and enzyme panel tests. Nevertheless, we would like to acknowledge gratitude to the Radiology and Biochemistry lab staff for their performance of the investigations.

\section{REFERENCES}

1. Adams LA, Talwalkar JA. Diagnostic evaluation of nonalcoholic fatty liver disease. J Clin Gastroenterol. 2006; 40 (Suppl 1): S34-S38.

2. Wieckowska A, Feldstein AE. Diagnosis of nonalcoholic fatty liver disease: invasive versus noninvasive. Semin Liver Dis. 2008; $28: 386-395$

3. Hernaez R, Lazo M, Bonekamp S, et al. Diagnostic accuracy and reliability of ultrasonography for the detection of fatty liver: a meta-analysis. Hepatology. 2011;54:1082-1090.

4. Vernon G, Baranova A, Younossi ZM. Systematic review: the epidemiology and natural history of nonalcoholic fatty liver disease and non-alcoholic steatohepatitis in adults. Aliment Pharmacol Ther. 2011; 34:274-285.

5. Chalasani N, Younossi Z, Lavine JE, et al. The diagnosis and management of non-alcoholic fatty liver disease: practice guideline by the American gastroenterological association, American association for the study of liver diseases, and American college of gastroenterology. Gastroenterology. 2012;142:1592-1609.

6. Aleksić V, Vučević D, Mladenović D, et al. Alcoholic liver disease/nonalcoholic fatty liver disease index. Eur J Gastroenterol Hepatol. 2013;25: 899-904.

7. Scherer A, Dufour JF. Treatment of Non-Alcoholic Fatty Liver Disease. Dig Dis. 2016;34:27-31.

8. U Satyanarayana, U chakrapani. Biochemistry. third edition. Kolkata: Arunabasen, 2005.

9. Dietrich ECF, Dietrich CF, Serra C, et al. EFSUMB European Course Book. Ultraschall der Medizin - Eur J Ultrasound. 2017; $33: 402$.

10. Toshikuni N, Tsutsumi M, Arisawa T. Clinical differences between alcoholic liver disease and nonalcoholic fatty liver disease. World J Gastroenterol. 2014;20:8393-8406.

11. Dasarathy S, Dasarathy J, Khiyami A, et al. Validity of real time ultrasound in the diagnosis of hepatic steatosis: a prospective study. J Hepatol. 2009;51:1061-1067.

12. Sevastianos VA, Dourakis SP. Alcoholic Liver Disease: A Clinical Review. J Nutr Food Sci. 2016; 06: 505

13. Poonam Mishra, MD, NilaRafiq M, and Zobair M. Younossi, MD, MPH, FACG F. Non-alcoholic fatty liver disease Nonalcoholic fatty liver disease. NC Med J. 2016;50: 1-5.

14. Mahaling DU, Basavaraj MM, Bika AJ. Comparison of lipid profile in different grades of non- alcoholic fatty liver disease diagnosed on ultrasound. Asian Pac J Trop Biomed. 2013;3: 907-912.

15. Lopez-Amador N, Nolasco-Hipolito C, Rojas-Jimeno MD, Carvajal-Zarrabal O. Liver enzymes in patients diagnosed with non-alcoholic fatty liver disease (NAFLD) in Veracruz: a comparative analysis with the literature. Clinical Investigation 2017;7(1):25-32.

16. Bhusal KR, Simkhada R, Nepal P. Lipid profile in different grades of Ultrasonic Non-Alcoholic Fatty Liver Disease. J Coll Med Sci. 2017;13:258-261.

17. Mittal A, Sathian B, Kumar A, Chandrasekharan N, Yadav S K.The Significance of Transaminases and Deritis Ratio for Predicting Alcoholic Liver Disease: A Hospital Based Comparative Study in Western Nepal. Nepal Journal of Epidemiology. 2010;1(1):33-37

18. Bishnu Jwarchan, Subita Lalchan, Anil Dhakal, Ramesh R Acharya. Comparison of liver enzymes and sonological grading in nonalcoholic fatty liver. Asian Journal of Medical Sciences. 2020;11(2):42-45

19. Ramesh, Krishnaswamy D, Indumati V, Vijay V, Rajeshwari Comparison of lipid profile and de-ritis ratio in ultrasound diagnosed non-alcoholic and alcoholic fatty liver disease. International Journal of Clinical Biochemistry and Research. 2016;3(4):438-441. 